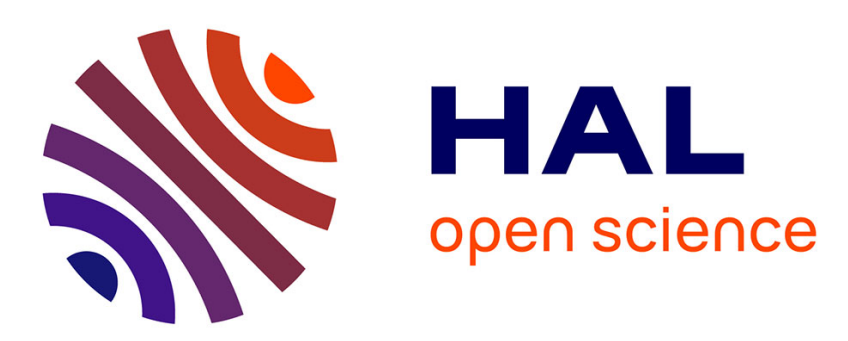

\title{
Integrated software for imaging data analysis applied to edge plasma physic and operational safety
} Vincent Martin, Victor Moncada, Gwenaël Dunand, Yann Corre, Elise Delchambre-Demoncheaux, Jean-Marcel Travere

\section{- To cite this version:}

Vincent Martin, Victor Moncada, Gwenaël Dunand, Yann Corre, Elise Delchambre-Demoncheaux, et al.. Integrated software for imaging data analysis applied to edge plasma physic and operational safety. 2010. hal-00536519

\section{HAL Id: hal-00536519 https://hal.science/hal-00536519}

Preprint submitted on 16 Nov 2010

HAL is a multi-disciplinary open access archive for the deposit and dissemination of scientific research documents, whether they are published or not. The documents may come from teaching and research institutions in France or abroad, or from public or private research centers.
L'archive ouverte pluridisciplinaire HAL, est destinée au dépôt et à la diffusion de documents scientifiques de niveau recherche, publiés ou non, émanant des établissements d'enseignement et de recherche français ou étrangers, des laboratoires publics ou privés. 


\title{
Integrated software for imaging data analysis applied to edge plasma physic and operational safety ${ }^{\text {th }}$
}

\author{
V. Martin ${ }^{\mathrm{a}}$, V. Moncada ${ }^{1}$, G. Dunand ${ }^{1}$, Y. Corre $^{\mathrm{a}}$, E. Delchambre ${ }^{\mathrm{a}}$, J.M. \\ Travere*,a \\ ${ }^{a}$ CEA, IRFM, F-13108 Saint Paul-lez-Durance, France. \\ ${ }^{b}$ Sophia Conseil Company, F-06560 Sophia Antipolis, France.
}

\begin{abstract}
Fusion tokamaks are complex devices requiring many diagnostics for real time control of the plasma and off-line physical analysis. In current tokamaks, imaging diagnostics have become increasingly used for these two purposes. Such systems produce a lot of data encouraging physicists to use shared tools and codes for data access and analysis. If general purpose software programs for data display and analysis are widely spread, a need exists in developing similar applications for quantitative imaging data analysis applied to plasma physic. In this paper, we introduce a new integrated software program, named WOLFF, dedicated to this task. The main contribution of this software is to gather under the same framework different functionalities for (1) data access and display, (2) signal, image, and video processing, and (3) quantitative analysis based on physical models.

After an overview of existing solutions for data processing in the field of plasma data, we present the WOLFF architecture and its currently implemented features. The capabilities of the software are then demonstrated through three applications in the field of physical analysis (heat and particle flux calculations) and tokamak operation (safety operation).
\end{abstract}

Key words: imaging diagnostic, software design, data analysis, plasma operation, image processing 


\footnotetext{
${ }^{\text {th }}$ WOLFF: An object-oriented platform dedicated to off-line quantitative analysis of multi-sensor imaging data

*Corresponding author. Tel: +33442256367

Email address: jean-marcel.travere@cea.fr (J.M. Travere)
} 
are given in section 5. Finally, future prospects are discussed in conclusion.

\section{Related Work}

Traditionally, physics experts working on a domestic fusion device develop their own solutions for data access, display and analysis according to their needs. If some of the solutions discussed below are optimized for general data display through a remote access, existing image-oriented programs are however too ad hoc to be interfaced with different data acquisition systems.

Among the popular signal-oriented programs shared in the fusion community, we can refer to JETDSP ${ }^{1}$. JETDSP is a distributed program to access, display, and analyze JET data in the IDL(C) environment that contributes to promote standardization [10]. Consequently, using this program for JET data analysis is a good solution to avoid conflicting interpretation of results that may occur when different tools are used. However, this program has a strong focus on signal processing, proposed image processing are very limited and few image/video formats are supported.

IRdisp [11] is a graphical user interface written in IDL developed at JET for infrared data display and analysis of recorded films acquired with the wide-angle view IR camera (KL7) and the divertor camera (KL3). This software has some advanced functionalities as heat flux calculation and image mapping onto 3D geometric model of the vessel inner components. It also provides some image processing functions as image noise filtering and camera shaking compensation based on edge detection. The main advantage of IRDisp is its integration into the JET environment. For instance, output files can be written in JET private format (PPF) for further treatments in JETDSP, and 3D model of plasma facing components can be loaded directly from JET CAD files (CATIA), without needing an external tool.

At Tore Supra, the acquired data from the six digital IR cameras are monitored so far with a dedicated software named ShotPlayer. ShotPlayer is multi-frame video player allowing, for one recorded film, a synchronized play of the six IR monitored views. A region analysis tool gives the possibility to extract the maximum temperature time-trace of one area in the image. Further analysis are mostly performed with MATLAB.

An alternative to the use of such dedicated software programs is then to develop its own routines using mainly MatLAB(C) or IDL. One interesting

\footnotetext{
${ }^{1}$ JETDSP for IDL homepage, http://www.jet.efda.org/expert/jetdsp/jetdsp.shtml.
} 


\begin{tabular}{lll}
\hline \hline \multicolumn{1}{c}{ Data Analysis Software } & \multicolumn{1}{c}{ Advantages } & \multicolumn{1}{c}{ Weaknesses } \\
\hline $\begin{array}{l}\text { interpreted languages with } \\
\text { image/signal processing } \\
\text { routines (e.g. } \begin{array}{l}\text { MATLAB, } \\
\text { IDL) }\end{array}\end{array}$ & $\begin{array}{l}\text { well-spread, available tool- } \\
\text { boxes, quick learn }\end{array}$ & $\begin{array}{l}\text { licensing policy, evolution } \\
\text { issues (version incompati- } \\
\text { bilities), speed limits }\end{array}$ \\
\hline $\begin{array}{l}\text { signal \& image processing } \\
\text { software (e.g. graphics edi- } \\
\text { tors) }\end{array}$ & optimized routines & $\begin{array}{l}\text { limited data management } \\
\text { possibilities }\end{array}$ \\
\hline & do exactly what users need & $\begin{array}{l}\text { code-sharing limitations, } \\
\text { leveloped in different } \\
\text { languages }\end{array}$ \\
\hline \hline
\end{tabular}

work based on some MATLAB's functions has been recently applied for invessel dust study in Tore Supra [12] by means of automatic image processing from CCD visible cameras. Such programming languages provide plenty of toolboxes (e.g. image processing) and rely on a large community of users and developers. Creation of graphical user interfaces, interfacing with others programs is even made easier with the help of wizards or tutorials. But these software programs raise some important issues. Indeed, a team working with MATLAB cannot reuse programs developed by others teams using IDL and vice versa. Moreover, these languages have no standard and the syntax may change and cause some backward compatibility issues. Finally, these interpreted language are quite slow, which makes heavy calculations taking a long time, thus not always suitable for repeated and automated computations.

Pros and cons of the discussed solutions are summarized in Table 1.

Table 1: Comparison table between different data analysis software programs with their main advantages and drawbacks.

Solutions deployed in other fusion devices like ASDEX equipped with imaging systems are not discussed here since they are very close to previously described ones or still in an early stage of development.

\section{WOLFF's software design}

We need a platform that integrates all analysis tools in the same framework and graphical user interface, and allows physicists to develop their own tools inside. First, this prevents from the parallel development of the same 
analysis code by different people. This would both result in decreasing the code quality and in wasting time. Second, this enables other people to reuse others tools for their own analysis. Finally, the use of common software enables all the developed tools to work together and thus to create a reference platform.

We also need a program that is reusable, modular and efficient; reusable because the needs may change, sometimes rapidly; modular because those changes must not disturb the structure of the program, and with high performance because of the large amount of data to analyze.

We decided to write WOLFF in $\mathrm{C}++$, since it is a multi-paradigm language controlled by a standard committee, it is one of the fastest language for complex computation, and also because many compilers are freely available for almost all operating systems [13]. Thus, physicists do not have to pay to get WOLFF working in their own environment. We made WOLFF opensource so that everyone may contribute by adding new modules or proposing changes to an existing one.

WOLFF takes advantage of the multiple paradigms offered by $\mathrm{C}++$, especially object-oriented programming and generic programming. On one hand, object-oriented programming allows to use design patterns [14] which encourage the use of high level abstractions. Those abstractions are crucial because they prevent major changes in the code due to minor modifications of the specifications. Indeed, a design pattern is a general and reusable solution to a frequently occurring problem in software design (as the factory pattern). Finally, design patterns enable reuse of others programmers' experience, speed up the development process, making the resulting code more robust than ad hoc solutions. For instance, the integration of a new data reading module does not affect the global system architecture. As seen in Figure 1, WOLFF is able to read infrared image data from Tore Supra and MAST in a transparent way for the user.

On the other hand, generic programming aims at finding abstract representations of efficient algorithms and data structures. The goal is to ease the re-use and the link of independent features, achieved by the template mechanism in $\mathrm{C}++$. As seen in Figure 2, the platform achieves therefore a high level of modularity. The source code makes heavy use of the Standard Template Library (STL), The Vigra library [15] and the Boost library [16] which provide high performance algorithms that can be applied on both vectors and images. Their speed is comparable to a direct RAM access through bit indexing, so the gained modularity has no consequences on the execution 


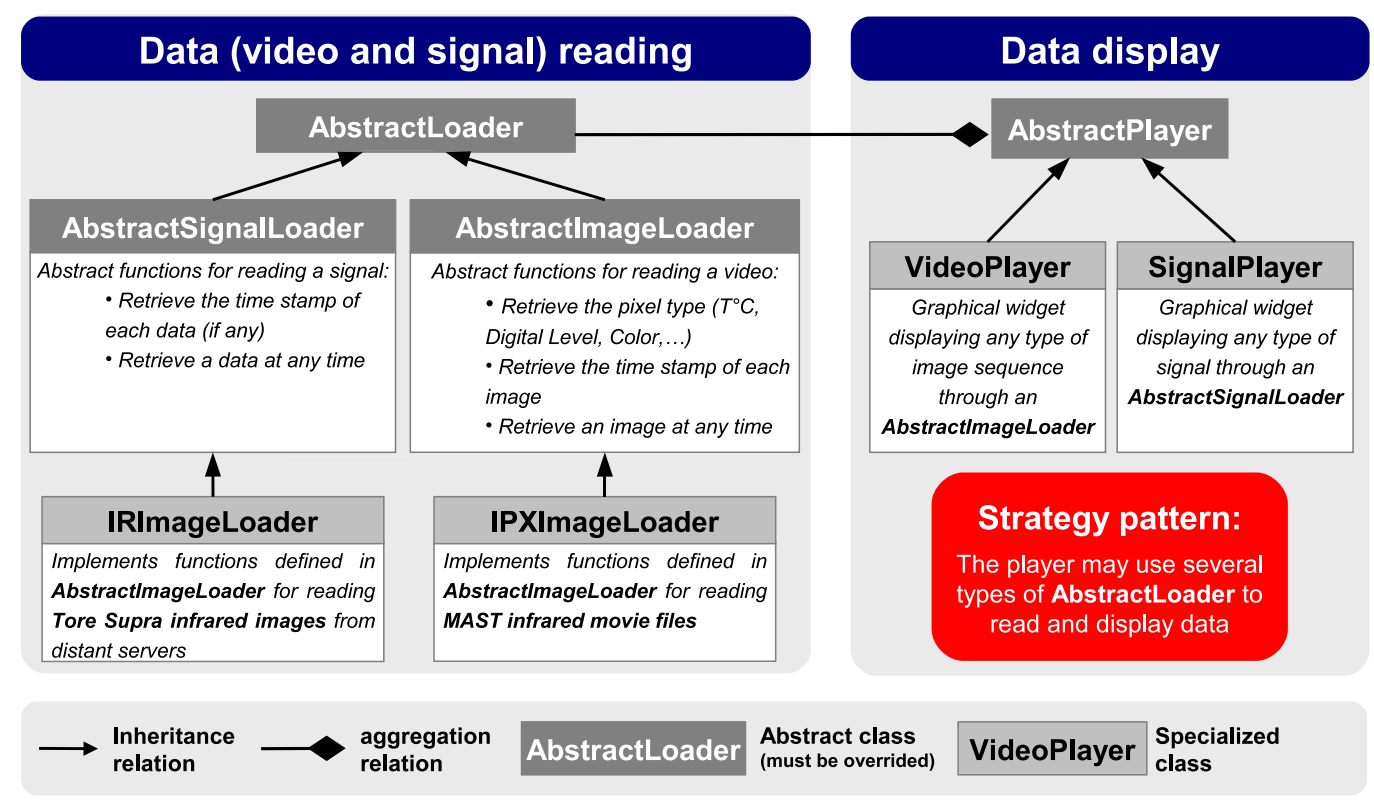

Figure 1: Example of abstraction defined in WOLFF making possible the reading of different types of data sources under the same framework.

time.

\section{WOLFF features}

The primary goal of this software is to efficiently read and display data from diagnostic databases in a graphical user interface. Currently, wolfF can open several types of multi-sensor data from Tore Supra data bases:

- image sequences or videos produced by imaging diagnostics (IR, visible) and stored in distant servers,

- signals produced by diagnostics or plasma control systems as plasma parameters, injected power levels, impurity level, etc.,

- data stored in local hard drives as images, video files, WOLFF files, predefined Regions of Interest (ROIs), or user scripts.

For the Tore Supra environment, WOLFF automatically uses a unique time base for each opened media (signal, image sequence and video) in order to 


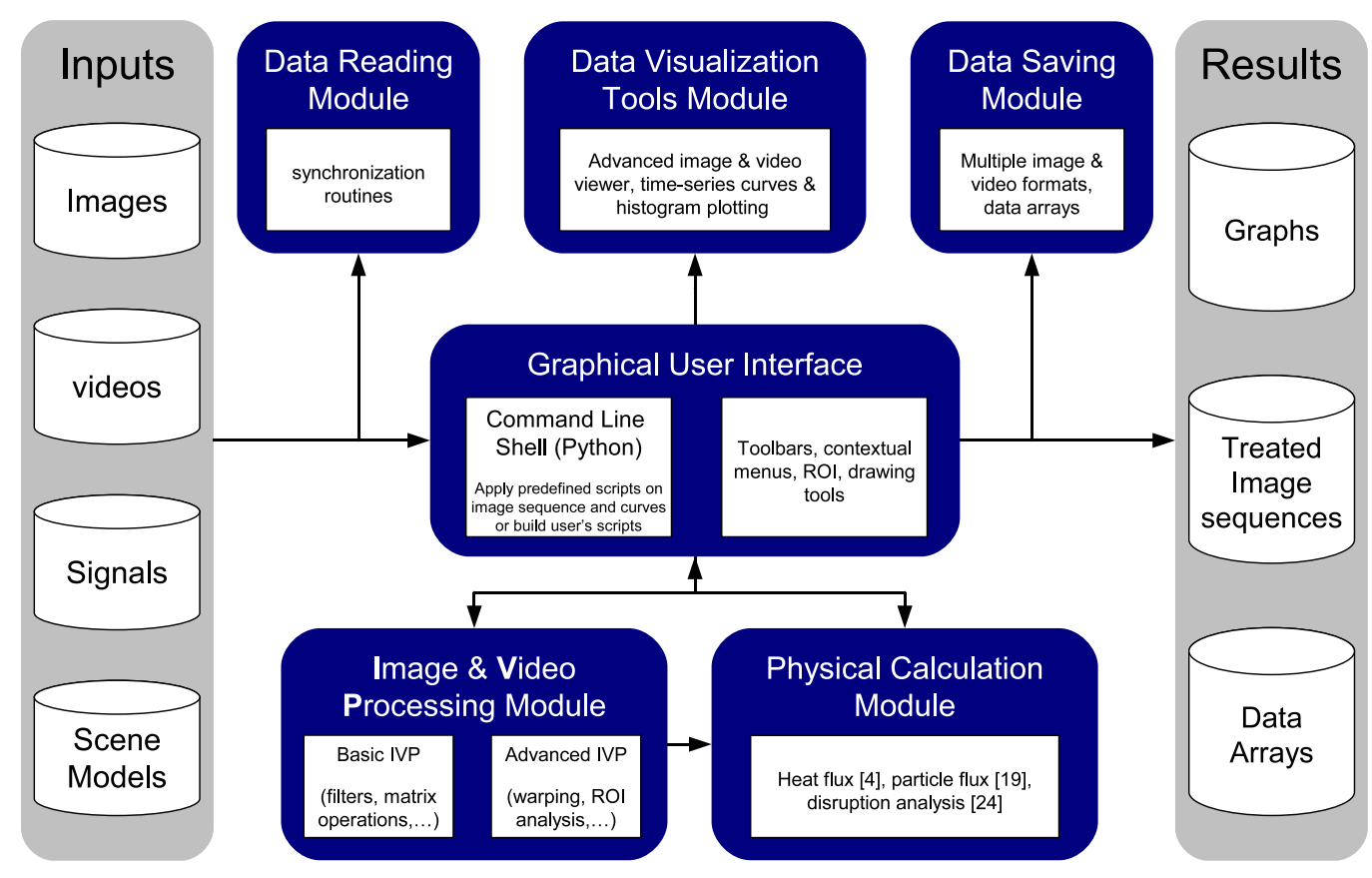

Figure 2: Schema of the modular architecture of WOLFF.

synchronize them all together. We can also mention that WOLFF is currently used at MAST (Mega Ampere Spherical Tokamak) for infrared data display and analysis.

\subsection{Data processing functions}

WOLFF provides an interactive tool for drawing ROI, allowing the user to create and modify several masks of different shapes (e.g.rectangles, circles, polygons). These masks are then used to process only interesting zones along an image sequence. The user can then extract and plot some features inside the defined ROI like region histograms or temporal evolution of pixel statistics as minimum, maximum, mean and variance values. It is also possible to extract a temperature profile from a line of pixels. Many image processing algorithms are implemented in WOLFF, reminding those available in MATLAB and image processing software programs as listed in Table 2.

From the most used features of WOLFF, we can refer to the warping tool. Digital image warping is a geometric transformation (scaling, rotation, etc.) applied locally or globally to a source image in space $(u, v)$ according to a 


\begin{tabular}{ll}
\hline \hline \multicolumn{1}{c}{ functions } & \multicolumn{1}{c}{ applications } \\
\hline $\begin{array}{l}\text { arithmetic operators for vector/matrix } \\
\text { manipulations }\end{array}$ & $\begin{array}{l}\text { time-series data analysis (e.g. image sub- } \\
\text { traction) }\end{array}$ \\
\hline $\begin{array}{l}\text { signal/image filtering, contour detection } \\
\text { (e.g., gaussian, sharpen, and Sobel filters) }\end{array}$ & signal/image de-noising and enhancing \\
\hline $\begin{array}{l}\text { geometric transformations like image } \\
\text { translation, reflection, rotation, and mor- } \\
\text { phological deformation [17] }\end{array}$ & image warping, morphological erosion \\
\hline $\begin{array}{l}\text { image segmentation based on thresholding } \\
\text { and background subtraction techniques }\end{array}$ & $\begin{array}{l}\text { object identification, overheating localiza- } \\
\text { tion }\end{array}$ \\
\hline \hline
\end{tabular}

Table 2: Overview of the different basic signal/image processing functions of wOLFF.

destination image in space $(x, y)$ as formalized in equation 1.

$$
I^{\prime}=\mathrm{f}(I) \quad \text { with } \quad \mathrm{f}: \underset{(u, v)}{\mathbb{R}^{2}} \longmapsto \underset{(x, y)}{\mathbb{R}^{2}}
$$

In practice, image warping is used to map two different images into a common geometry. The mapping may be derived given a model of the geometric distortions of a system, but more typically the mapping is inferred from a set of corresponding points (called control points) in the source and destination images.

The computation of the mapping function $\mathrm{f}$ is usually achieved by interpolation functions. In our approach, we use a weighted linear interpolation based on the $k$ nearest control points from each pixel in the source image.

Let $(u, v)$ denote the position of a pixel $p$ in the original image $I$ and $(x, y)$ its position in image $I^{\prime}$. Let $\left\{p_{1}\left(u_{1}, v_{1}\right), \ldots, p_{k}\left(u_{k}, v_{k}\right)\right\}$ the set of the $k$ nearest control points to $p$ defined by the user on both $I$ and $I^{\prime}$. The mapping of the position of $p$ from $I$ to $I^{\prime}$ is such as:

$$
\forall p(u, v) \in I,\left\{\begin{array}{l}
x=\mathrm{f}_{x}(u)=u+\frac{1}{S D} \sum_{i=1}^{k} \mathrm{~d}\left(p, p_{i}\right) \cdot\left(u-u_{i}\right) \\
y=\mathrm{f}_{y}(v)=v+\frac{1}{S D} \sum_{i=1}^{k} \mathrm{~d}\left(p, p_{i}\right) \cdot\left(v-v_{i}\right)
\end{array}\right.
$$

where $S D=\sum_{i=1}^{k} \mathrm{~d}\left(p, p_{i}\right)$ and $\mathrm{d}(.,$.$) is the euclidean distance between$ two points defined by:

$$
\forall p, q \in \mathbb{R}^{2}, \quad \mathrm{~d}(p, q)=\sqrt{\left(x_{p}-x_{q}\right)^{2}+\left(y_{p}-y_{q}\right)^{2}}
$$


In the field of tokamak application, this technique is very useful to map an image acquired with a visible or IR imaging diagnostic onto the geometry of a real model derived from in-vessel CAD files or represented by in situ picture. A good illustration of this technique is given in section 5.1 for heat flux calculation.

\subsection{User's interface}

In order to keep the software both user-friendly and flexible, algorithms can be applied in two different ways. Routinely used operations are directly accessible via icons in tool bars or in scrolling/contextual menus. User specific treatments can be loaded, modified, applied and saved via a command line shell. Indeed, WOLFF embeds a Python ${ }^{\mathrm{TM}}$ shell (Python is an interpreted programming language quite similar to IDL) providing all common Python tools and image processing functions. This way, a user can implement its own chain of processes and apply it on a single image or on an image sequence. For example, the set of treatments shown below is used to automate the detection of hot spot observed prior to radiative disruptions on Tore Supra limiter [18] and localized on the high field side of the limiter. extract the boundaries of an image:

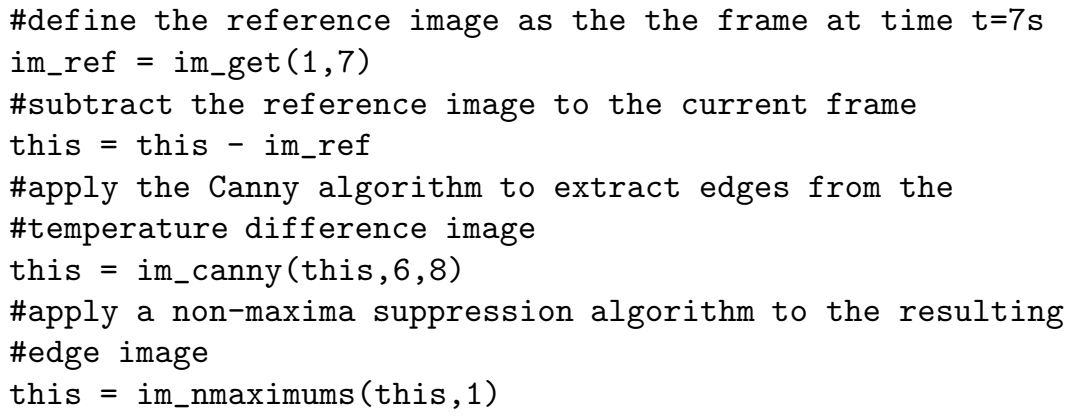

As a real live example, figure 3 shows how this image processing chain can be used in a WOLFF session. The lower panel shows the infrared image sequence of the Tore Supra limiter for the plasma discharge \#38425 at time $\mathrm{t}=7 \mathrm{~s}$ (disruption at $8.38 \mathrm{~s}$ ). The goal is then to automatically extract the hot spot appearing on thick carbon redeposition zones just before the disruption. To this end, the user has defined a small script using the Python shell visible on the right panel. The procedure consists in first subtracting to the current frame a reference image taken few frames before (i.e. during steady-state). The temperature difference image is then filtered with the Canny edge detector [19]. The result is visible on the top panel (white circle) and is directly 
superimposed on the current frame (time $\mathrm{t}=8.2 \mathrm{~s}$ ). This procedure can be easily repeated over several disruptive discharges to automatically extract useful information on precise location and shape of such hot spots.

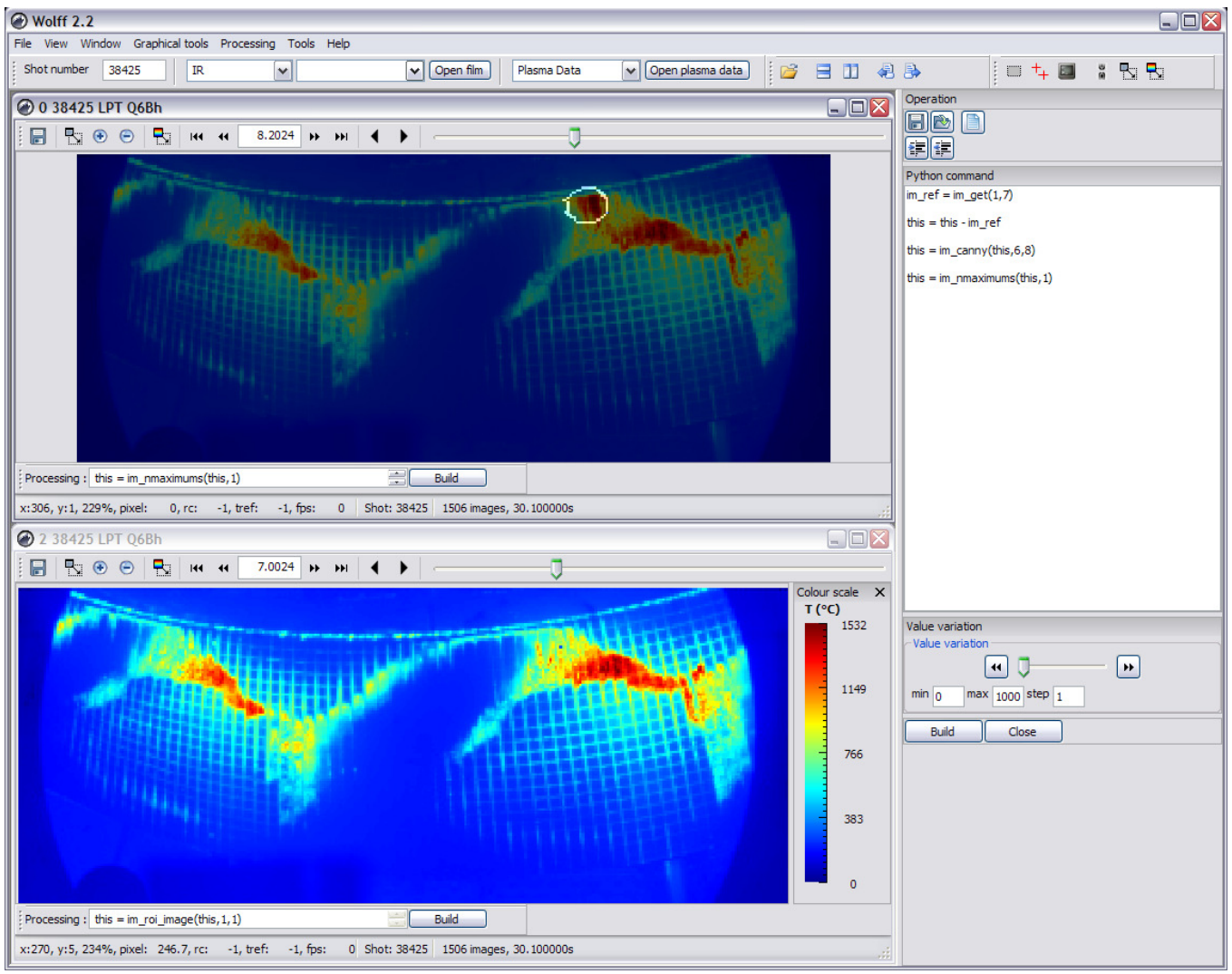

Figure 3: Real live example of using different features of WOLFF (image processing using Python script, image superimposition) for infrared video analysis. Here the goal is to highlight hot spot patterns observed on the limiter just before a radiative disruption (see [18] for details).

The Python shell also provides algorithms for the analysis of monodimensional signals, and useful functions for I/O operations like opening, saving and closing any kind of media. Creating and embedding a new filter or a new algorithm in python syntax thus becomes an easy task for physicists. 


\subsection{Quantitative analysis using physical models}

Currently, the most complex function is a heat flux calculation algorithm based on the quadrupole method using the infrared diagnostic [20]. This functionality gives the possibility to create dynamic heat flux cartographies, useful for physical analysis (see section 5.1 for details).

\section{Applications}

WOLFF has been successfully used by physicists for different quantitative analysis related to machine protection issues. The two main analysis carried out in Tore Supra are heat flux calculation using infrared imaging and calculation of the carbon erosion (particle flux) on the TPL using visible imaging. PFCs monitoring is also of a crucial help during plasma operation, especially at high power level. The goal is to extract from imaging data three parameters: localization of the overheating region, identification of the underlying component, and characterization of the thermal event trough its temporal evolution. Such information is then used by plasma operators for the estimation of the ongoing safeness level. In this section, we relate these three case studies of using WOLFF as an integrated software program for advanced physical analysis and for operational safety.

\subsection{Heat flux calculation}

WOLFF integrates a heat flux computation tool able to estimate the heat flux deposit on Tore Supra TPL in order to perform a heat balance. This tool uses several functions: morphological deformation of infrared images to work in drawing office geometry (physical components are then well-identified), definition of the regions corresponding to the TPL's tiles, and extraction of the maximum and mean temperatures inside these regions. More precisely, we use the quadrupole model [20] to solve the fundamental heat transfer equation. In our case, the deposited heat flux is supposed to be homogeneous over the tile surface. The heat diffusion is then only in the depth direction making the problem mono-dimensional. The quadrupole model requires for each tile of the TPL the extraction of the average temperature. To this end, the infrared image (Figure 4(a)) must be mapped onto the real geometry of the TPL represented by a grid (Figure 4(b)) in order to identify each of the TPL tile. Each cell of the grid represents a tile representing a ROI. The mapping is achieved using the warping tool of WOLFF based on elastic deformation 
algorithms. The user's task consists in setting control points on the two images (Figure 4(c) and Figure 4(d)) by using the footprint of the components (waffle structure) visible in the infrared image. The superimposition of the warped infrared image with the image in the real geometry helps to check the quality of the warping. The poorly attached carbon deposits between tile gaps lead to higher local temperature [21] than the tile surface. In order to eliminate this deleterious effect for heat flux calculation, a morphological erosion is applied to each ROI to take into account only the central pixels of a tile (Figure 4(e)). Finally, temporal evolutions of mean and maximum temperatures are saved for each ROI and given as input to the heat flux algorithm integrated into WOLFF. An illustration of the first steps leading to the heat flux estimation is given in Figure 4.

Towards temperature measurement used for heat flux calculation, one may take attention to error bar using image data. This is indeed in an important issue since the estimation of the error bar introduced for each pixel is mainly dependent of the measurement system calibration. Currently, for Tore Supra infrared diagnostic, the calibration function is already taken into account into the temperature conversion function used in WOLFF. Upgraded version of the software could easily integrate this calibration function as a separated module thus enabling further error bar estimation.

\subsection{Particle flux calculation}

The second application which takes advantages of the warping tool and the image superimposition tool of WOLFF is the determination of the carbon erosion [22] using visible imaging. In addition to infrared endoscopes, a visible camera coupled with a set of four optical fibres linked to a spectrometer looks simultaneously the $C D, H_{\alpha}$, and $C I I$ emission [23] from the same region of the TPL. This system can be coupled with a high infrared resolution camera in order to superimpose any type of 2D mapping such as atomic/molecular emission on heat load. This multi-spectral approach coupled with infrared thermography diagnostic is carried out for three reasons: 1) to de-correlate atomic/molecular photons from Planck emission, 2) to characterize chemical erosion with surface temperature and 3) to study the contribution of gaps in the carbon erosion/migration process. Finally, the particle flux is obtained from the absolutely calibrated brightness using the standard procedure described in [22]. 


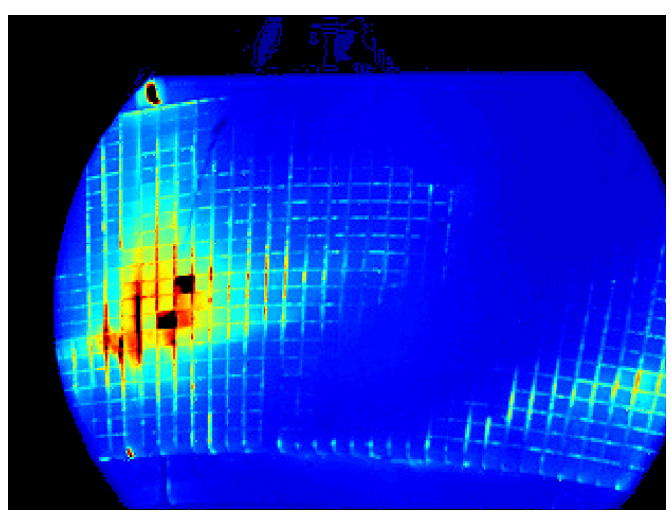

(a) IR view of the TPL

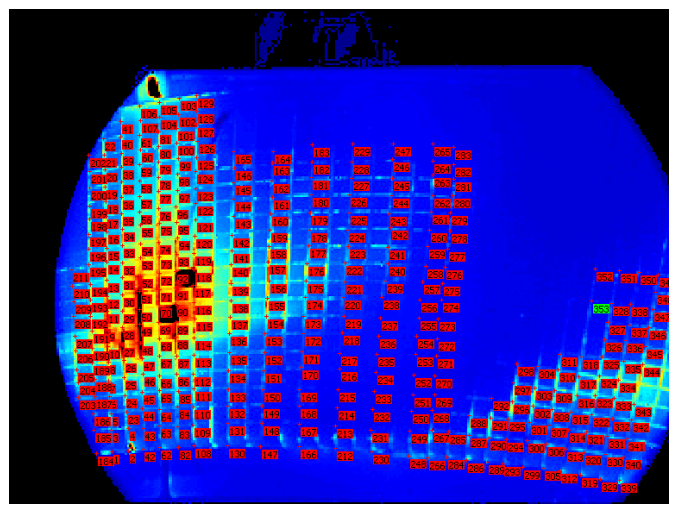

(c) Control points set on (a)

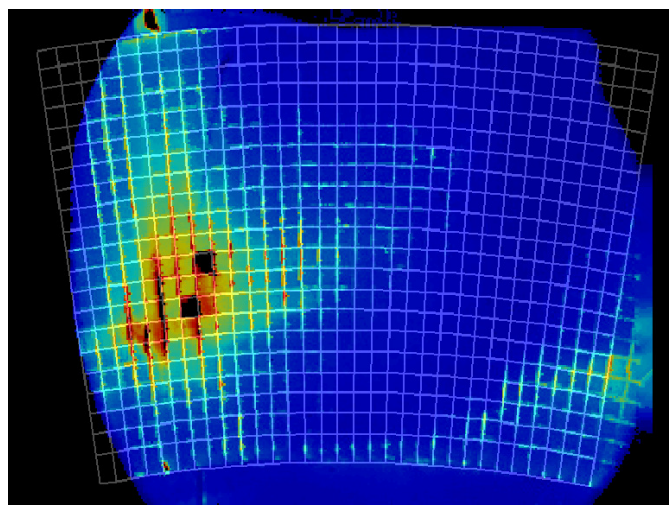

(e) Warped IR view superimposed with (b) (f) Temperature cartography using eroded (b) masks

Figure 4: Using WOLFF for temperature cartography of the Tore Supra's TPL tiles without deleterious waffle effect.

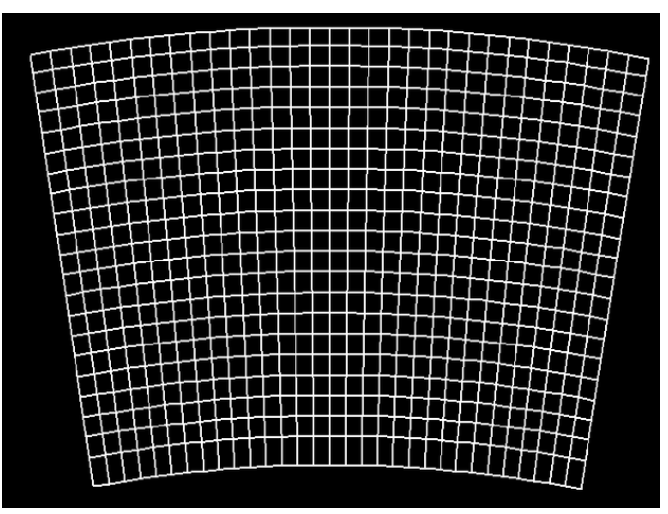

(b) Model of the TPL's real geometry

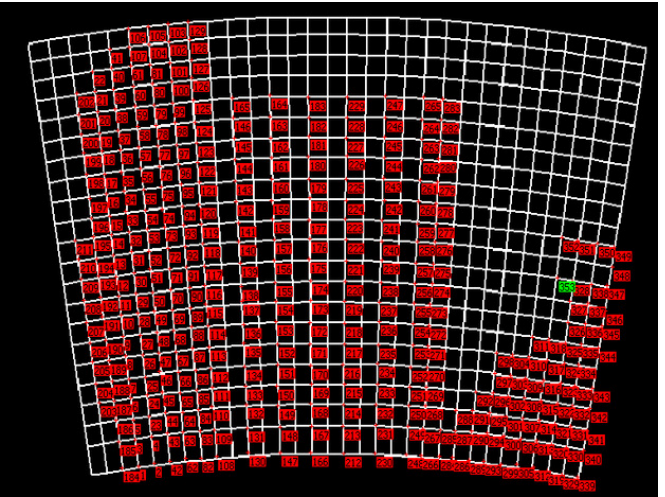

(d) Control points set on (b)

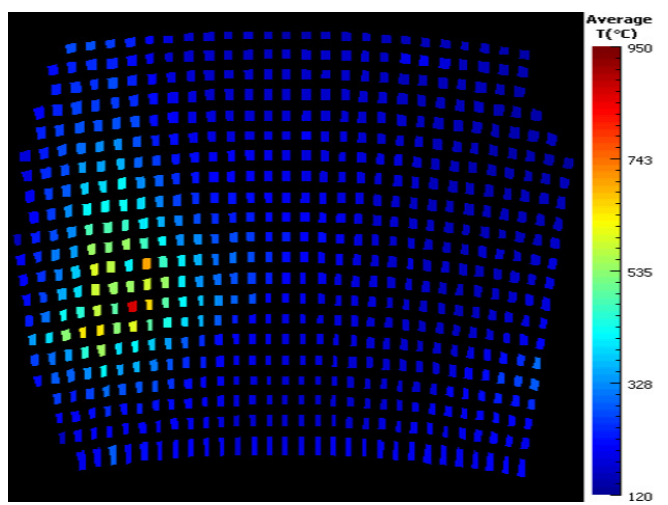

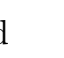


5.3. Computer-aided infrared image interpretation for plasma safety operation

If the quantitative physical analysis of plasma data is mostly achieved after plasma operation, it is sometime necessary to have a quick interpretation of data between two plasma discharges. For instance, several plasma parameters as injected power levels must be checked to diagnose an abnormal thermal event observed in the infrared images. As seen in Figure 6, the user interface of WOLFF offers a global view of different signals with synchronization and temporal marks making easier the abnormal event evidence and so the infrared data interpretation. A second helpful feature is the superimposition between infrared images onto the real geometry of the monitored PFCs allowing an accurate identification of the overheating zones before a physicist interpretation. Finally, WOLFF offers easy and quick functions to plot temporal evolutions of different ROI in order, for instance, to compare suspicious heating zones.

Figure 5 shows the localization of four different hot spots identified during previous physical analysis and described below:

HS1 (local RF sheath effect) : Localized on the top left corner of the Faraday screen (made of stainless steel with $B_{4} C$ coating), this hot spot is suspected to be due to local RF electrical field carrying an enhanced power onto the antenna as explained in [24]. The deposited power causes hot spots with risk of melting and bursts of metallic impurities.

HS2 (accelerated electrons from lower hybrid) : This hot spot is localized on the left side protection (made of graphite tiles). It is suspected to be due to electrons accelerated in the near field of the lower hybrid launcher which is magnetically connected to the ICRH antenna as explained in [25].

HS3 (fast ion losses) : This hot spot is localized both on the side protection and the vertical edge of the Faraday screen. It might be caused by fast ions losses created during ICRH hydrogen minority heating scheme as explained in [26]. In Tore Supra these losses are an important cause of concern for the long pulse capability at high RF power levels due to high magnetic ripple (see [27]).

HS4 ( $B_{4} C$ flakes) : The hot spot has a small size and is localized on the vertical edge of the Faraday screen. It is due to the flaking of the $B_{4} C$ 


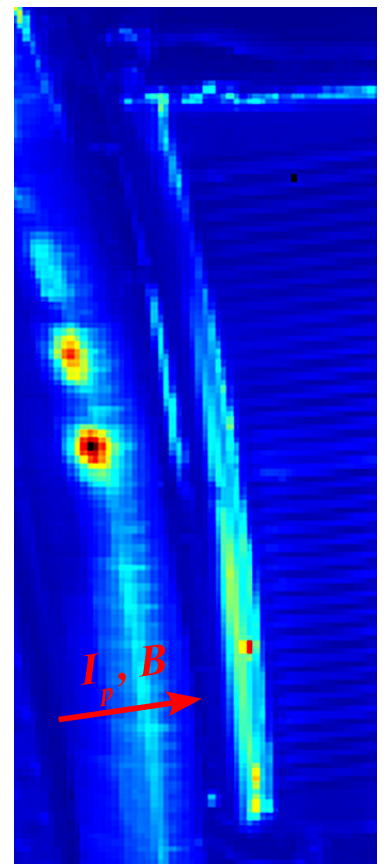

(a) Infrared image of the heating antenna (left hand side).

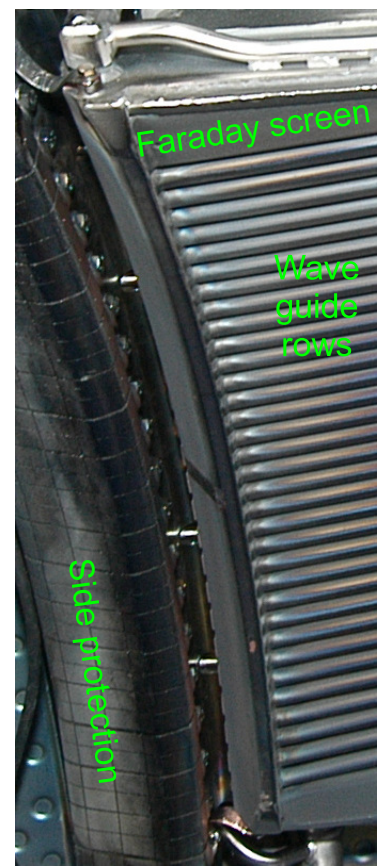

(b) Visible view of the heating antenna (left hand side).

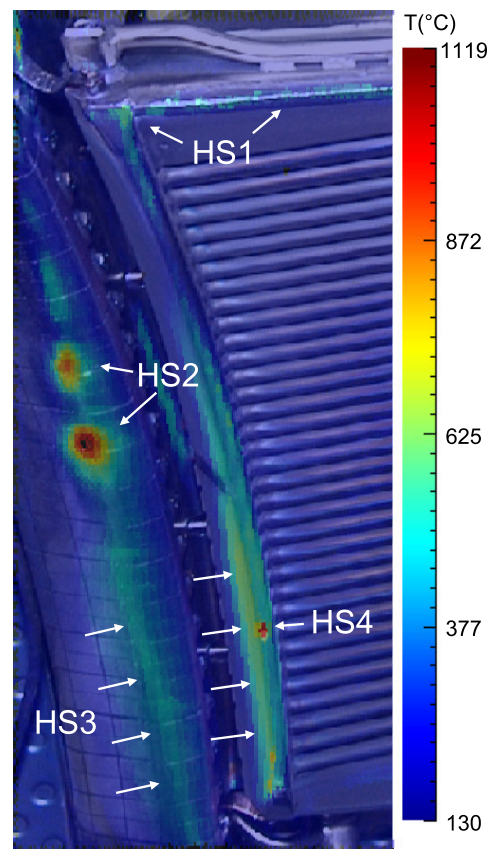

(c) Visible view superimposed with the warped infrared image highlighting Hot Spots (HS) on PFCs.

Figure 5: Using WOLFF during plasma operation to localize precisely the heating areas on PFCs.

coating consequently to the heating caused by fast ion losses as shown in [28]. Temperature may overpass the acceptable threshold without apparent risk of damage.

\section{Conclusion}

In this paper, we have presented a new integrated software program developed at Tore Supra and dedicated to the quantitative analysis of multi-sensor data produced by different diagnostics. Compared to existing solutions, the main advantage of WOLFF is to gather under the same graphical user interface common routines for data access, manipulation, and visualization as well as specific functions for physic analysis purposes with a strong emphasis on image processing. WOLFF is daily used at Tore Supra and MAST by 


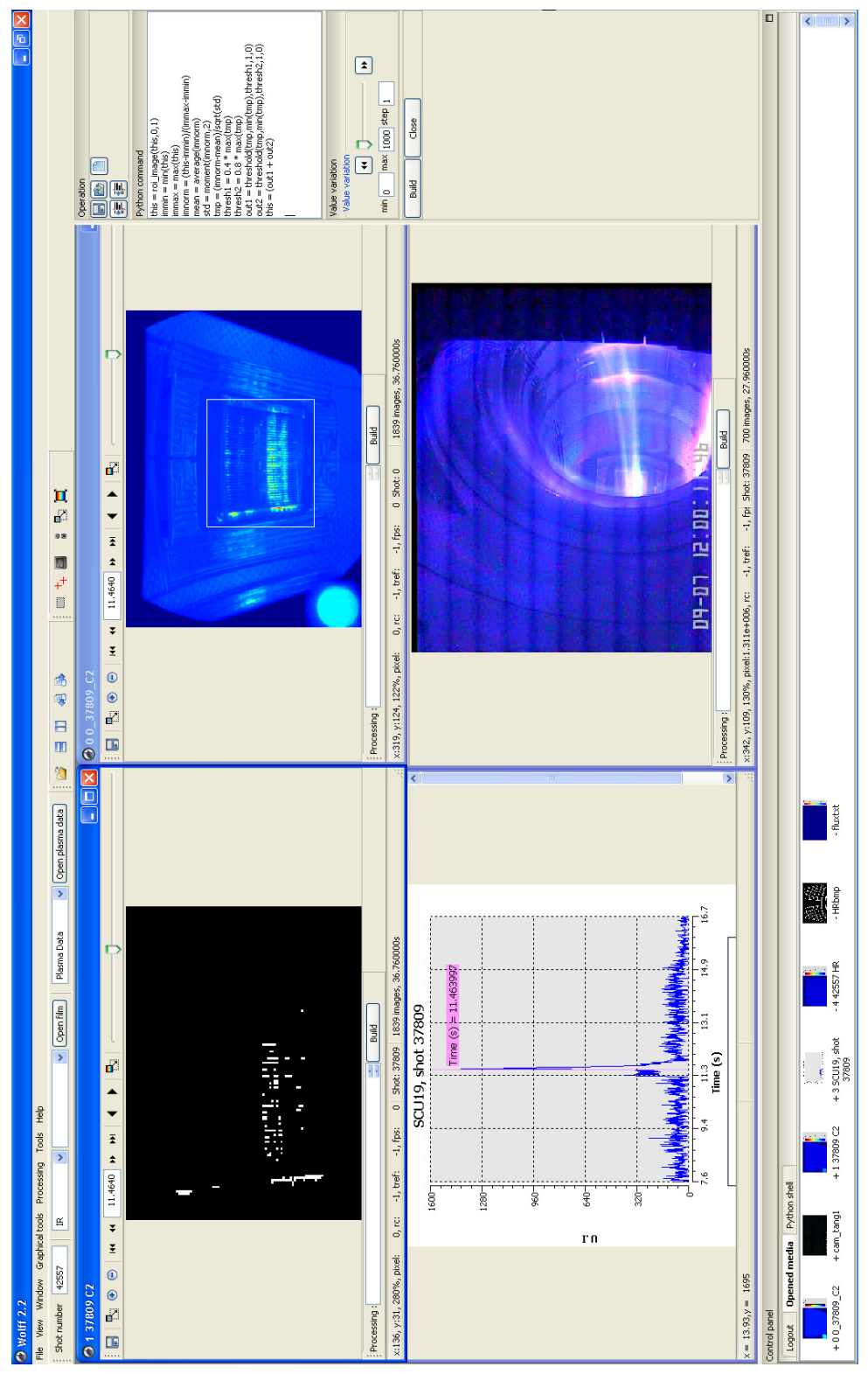

Figure 6: An example of a WOLFF session during off-line plasma analysis. The main window displays LHCD IR view (top right), equatorial visible view (bottom right), and copper signal from the UV spectrometer (bottom left). All data are synchronized in time. The user has defined a ROI on the IR view and has written a script composed of a chain of image processing visible on the right part of the main window. The result of this treatment (adaptive image thresholding to detect abnormal overheating pixels) is displayed on the top left frame (black pixel corresponds to image background). 
physicists for both quantitative physical analysis and safe plasma operation. Moreover, the modular design of its architecture eases the development and the integration of new algorithms according to the physicist requests.

Future long pulse reactors like ITER will produce a very large amount of data at each pulse, and will be the place for new physic experimentations. These two challenges imply new tools for the understanding of plasma-wall interactions. Such computer-aided system might be one part of the solution for the data management and processing issues related to imaging systems.

\section{Acknowledgment}

This work, supported by the European Communities under the contract of Association between EURATOM and CEA, was carried out within the framework of the European Fusion Development Agreement. The views and opinions expressed herein do not necessarily reflect those of the European Commission.

\section{References}

[1] J.-M. Travere and Tore Supra Team, In-vessel components imaging systems: From the present experience towards iter safe operation, Fusion Engineering and Design 84 (7-11) (2009) 1862 - 1866.

[2] D. Guilhem, et al., Tore-supra infrared thermography system, a real steady-state diagnostic, Fusion Engineering and Design 74 (1-4) (2005) $879-883$.

[3] P. Moreau, et al., RF heating optimization on Tore Supra using feedback control of infrared measurements, Fusion Engineering and Design 82 (514) (2007) $1030-1035$.

[4] S. Carpentier, et al., Study of heat flux deposition on the limiter of the tore supra tokamak, Journal of Nuclear Materials 390-391 (2009) 955 958.

[5] G. Arnoux, and JET EFDA contributors, Plasma-wall heat loads in iterlike advanced tokamak scenarios on jet, in: 34th EPS Conf. on Plasma Physics, Vol. 31(F), Warsaw, Poland, 2007, p. 1023. 
[6] R. Robb, D. Hanson, R. Karwoski, A. Larson, E. Workman, M. Stacy, Analyze - a comprehensive, operator-interactive software package for multidimensional medical image display and analysis, Computerized Medical Imaging and Graphics 13 (6) (1989) 433-454.

[7] J. Travere, P. Allain, B. Landeau, An object-oriented approach for quantitative interpretation of multimodal images, in: Engineering in Medicine and Biology Society, 1992. Vol.14. Proceedings of the Annual International Conference of the IEEE, Vol. 3, 1992, pp. 938-940.

[8] B. Diallo, F. Dolidon, J. Travere, B. Mazoyer, VoxeLine: a software program for 3D real-time visualization of biomedical images, Computerized Medical Imaging and Graphics 22 (4) (1998) 275-289.

[9] A. Hajdu, J. Kormos, Z. Lencse, L. Trón, M. Emri, The medip-platform independent software system for medical image processing project, Journal of Universal Computer Science 12 (9) (2006) 1229-1239.

[10] W. Suttrop, D. Kinna, J. Farthing, O. Hemming, J. How, V. Schmidt, Remote participation at jet task force work: users' experience, Fusion Engineering and Design 60 (3) (2002) 459 - 465.

[11] G. Arnoux, Irdisp: a graphical user interface for infrared data analysis on jet, Tech. rep., JET EFDA (2007).

[12] S.-H. Hong, C. Grisolia, P. Monier-Garbet, Development of automatic data extraction technique from visible ccd images for in-vessel dust study in tore supra, Plasma Physics and Controlled Fusion 51 (7) (2009) 075013.

[13] H. Sutter, Exceptional C++: 47 engineering puzzles, programming problems, and solutions, Addison-Wesley Longman Publishing Co., Inc., Boston, MA, USA, 2000.

[14] E. Gamma, R. Helm, R. Johnson, J. Vlissides, Design patterns: elements of reusable object-oriented software, Addison-Wesley Professional, Reading, MA, 1995.

[15] U. Köthe, STL-Style Generic Programming with Images, C++ Report Magazine 12 (1) (2000) 24-30. 
[16] D. Abrahams, R. W. Grosse-Kunstleve, Building hybrid systems with boost. python, $\mathrm{C} / \mathrm{C}++$ Users Journal 21 (7).

[17] G. Wolberg, Digital Image Warping, IEEE Computer Society Press, Los Alamitos, CA, USA, 1994.

[18] A. Ekedahl, et al., Analysis of radiative disruptions in rf-heated tore supra plasmas using infrared imaging, Journal of Nuclear Materials 390391 (2009) $806-809$.

[19] F. J. Canny, A Computational Approach to Edge Detection, IEEE Trans. on Pattern Analysis and Machine Intelligence 8 (6) (1986) 679698.

[20] J.-L. Gardarein, et al., Thermal quadrupoles approach for twodimensional heat flux estimation using infrared and thermocouple measurements on the jet tokamak, International Journal of Thermal Sciences 48 (1) (2009) $1-13$.

[21] R. Mitteau, et al., Evaluation of the growth of carbonaceous deposit in steady state tore supra using infrared thermography, Nuclear Fusion 46 (3) (2006) S49.

[22] E. Delchambre, et al., Characterization of the carbon erosion on the limiter of tore supra, Journal of Nuclear Materials 390-391 (2009) 65 67.

[23] E. Delchambre, et al., Multi spectral imaging system as a tool for erosion characterization, in: IAEA Workshop Challenges in plasma spectroscopy for future fusion research, 2008.

[24] L. Colas, et al., Hot spot phenomena on Tore Supra ICRF antennas investigated by optical diagnostics, Nuclear Fusion 43 (2003) 1-15.

[25] M. Goniche, et al., Enhanced heat flux in the scrape-off layer due to electrons accelerated in the near field of lower hybrid grills, Nuclear Fusion 38 (6) (1998) 919-937.

[26] V. Basiuk, et al., Ripple losses during icrf heating in tore supra, Nuclear Fusion 44 (1) (2004) 181-192. 
404

[27] A. Ekedahl, et al., Operational limits during high power long pulses with radiofrequency heating in tore supra, Nuclear Fusion 49 (9).

[28] M. Chatelier, Integration of high power, long pulse operation in tore supra in preparation for iter, Nuclear Fusion 47 (10) (2007) S579-S589. 